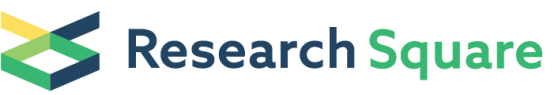

\section{Hepatic serine and lipid metabolism drive diabetic peripheral neuropathy}

\section{Christian Metallo ( $\nabla$ metallo@salk.edu )}

metallo@salk.edu

\section{Michal Handzlik}

University of California, San Diego https://orcid.org/0000-0002-4653-1902

\section{Jivani Gengatharan}

The Salk Institute for Biological Studies

\section{Katie Frizzi}

UCSD

\section{Grace McGregor}

The Salk Institute for Biological Studies

\section{Cameron Martino}

University of California, San Diego https://orcid.org/0000-0001-9334-1258

\section{Gibraan Rahman}

University of California San Diego

\section{Antonio Gonzalez}

University of California, San Diego

\section{Ana Moreno}

University of California San Diego

\section{Courtney Green}

The Salk Institute for Biological Studies

\section{Terry Lin}

The Salk Institute for Biological Studies

\section{Yoichiro Ideguchi}

Scripps Research

\section{Regis Fallon}

Lowy Medical Research Institute https://orcid.org/0000-0002-2391-2534

\section{Amandine Chaix}

University of Utah https://orcid.org/0000-0001-7007-197X

\section{Martina Wallace}

University College Dublin https://orcid.org/0000-0001-8272-5678

\section{Prashant Mali}

University of California San Diego

\section{Marin Gantner}


Lowy Medical Research Institute

Nigel Calcutt

University of California San Diego

Robin Knight

University of California, San Diego https://orcid.org/0000-0002-0975-9019

Biological Sciences - Article

Keywords:

Posted Date: January 14th, 2022

DOI: https://doi.org/10.21203/rs.3.rs-1189916/v1

License: (c) (1) This work is licensed under a Creative Commons Attribution 4.0 International License. Read Full License 
Title: Hepatic serine and lipid metabolism drive diabetic peripheral neuropathy

Michal K. Handzlik ${ }^{1,2}$, Jivani M. Gengatharan ${ }^{1,2}$, Katie E. Frizzi ${ }^{3}$, Grace H. McGregor ${ }^{1,2}$, Cameron Martino ${ }^{4,5,6}$, Gibraan Rahman ${ }^{4,5}$, Antonio Gonzalez ${ }^{4}$, Ana M. Moreno ${ }^{2}$, Courtney R. Green ${ }^{1,2}$, Terry Lin ${ }^{7}$, Yoichiro Ideguchi ${ }^{8}$, Regis J. Fallon ${ }^{9}$, Amandine Chaix ${ }^{10}$, Martina Wallace ${ }^{11}$, Prashant Mali $^{2}$, Rob Knight ${ }^{2,4,5,6}$, Marin L. Gantner ${ }^{9}$, Nigel A. Calcutt ${ }^{3}$, and Christian M. Metallo ${ }^{1,2, \dagger}$

${ }^{1}$ Molecular and Cell Biology Laboratory, The Salk Institute for Biological Studies, La Jolla, CA, USA

${ }^{2}$ Department of Bioengineering,

${ }^{3}$ Department of Pathology, School of Medicine, University of California San Diego, La Jolla, CA, USA

${ }^{4}$ Department of Pediatrics, School of Medicine,

${ }^{5}$ Bioinformatics and Systems Biology Program,

${ }^{6}$ Center for Microbiome Innovation, University of California San Diego, La Jolla, CA, USA.

${ }^{7}$ Regulatory Biology Laboratory, The Salk Institute for Biological Studies, La Jolla, CA, USA

${ }^{8}$ Scripps Research, La Jolla, CA, USA

${ }^{9}$ Lowy Medical Research Institute, La Jolla, CA, USA

${ }^{10}$ Department of Nutrition and Integrative Physiology, University of Utah, Salt Lake City, UT, USA

${ }^{11}$ School of Agriculture and Food Science, University College Dublin, Dublin, Ireland

† Email: metallo@salk.edu 
Type 2 diabetes represents a disease spectrum in which chronic metabolic dysfunction damages multiple organ systems including liver, kidneys, and peripheral nerves ${ }^{1,2}$. While onset and progression of these co-morbidities are linked with insulin resistance, hyperglycaemia and dyslipidemia ${ }^{3-7}$, aberrant amino acid metabolism also contributes to pathogenesis of diabetes and potentially its complications ${ }^{8-10}$. Serine and glycine are closely related non-essential amino acids $^{11,12}$ that are consistently reduced in patients with metabolic syndrome ${ }^{10,13-16}$, but the mechanistic drivers of serine deficiency and the downstream metabolic and phenotypic consequences remain unclear. Low systemic serine (and glycine), a serine-opathy, is also emerging as a hallmark of macular and peripheral nerve disorders, correlating with impaired visual acuity and peripheral neuropathy $(P N)^{17-19}$. Here we demonstrate that aberrant serine homeostasis in the liver drives serine and glycine deficiencies in genetically obese and hyperglycaemic mice. This serine-opathy can be diagnosed with a serine tolerance test that quantifies systemic serine disposal. Mimicking these metabolic alterations via dietary serine/glycine restriction together with high fat intake dramatically accelerates thermal hypoalgesia in mice and reduces epidermal sensory nerve density, which are accompanied by extensive sciatic nerve lipid remodeling. These phenotypes were subsequently normalized by myriocin, linking serine-associated PN with sphingolipid metabolism. These findings identify systemic serine deficiency and dyslipidemia as novel risk factors for PN that may be exploited therapeutically.

\section{Aberrant serine and glycine metabolism is associated with diabetic peripheral neuropathy}

Low circulating serine and glycine are hallmarks of metabolic disorders ${ }^{8,10,14}$, yet how and why obesity and type 2 diabetes (T2D) impact serine and glycine metabolism across tissues is poorly defined. To explore this relationship in more detail, we quantified serine, glycine, and methionine in an established mouse model of morbid obesity and hyperglycaemia driven by leptin-receptor deficiency, $d b / d b$ mice on Black Kaliss (BKS) background, comparing results to age-matched wild-type (WT) controls. Relative to WT, fasting $d b / d b$ mice exhibited a $\sim 30 \%$ reduction in serine levels within the liver and kidney (Fig. 1a, Extended Data Fig. 1a). Glycine abundance, which is $5-10$-fold greater than serine in tissues, was also reduced by $\sim 30-50 \%$ in liver, kidney, inguinal white adipose tissue (iWAT), and plasma (Fig. 1a, Extended Data Fig. 1a-c). Methionine is linked with serine metabolism via the one-carbon pathway, and its levels were also reduced in liver, iWAT, and plasma (Fig. 1a, Extended Data Fig. 1b-c). Collectively, these results indicate that obesity/diabetes is associated with lower levels of serine and glycine in tissues important for glucose and lipid homeostasis.

Mammals obtain serine and glycine from the diet or via de novo synthesis from glucose. The liver is a major site for post-prandial metabolism of carbohydrates, lipids, and amino acids such as serine and glycine, and methionine. To better understand the mechanistic basis of reduced hepatic serine and glycine levels in $d b / d b$ mice, we quantified expression of genes associated with serine, glycine, and one carbon (SGOC) metabolism (Fig. 1b). Key enzymes responsible for serine catabolism or disposal were significantly upregulated in $d b / d b$ mice, including serine dehydratase (Sds), serine hydroxymethyltransferase 1 (Shmt1), and Shmt2. Two components of the glycine cleavage system, glycine decarboxylase (G/dc) and dihydrolipoamide dehydrogenase $(D / d)$, as well as 10 -formyltetrahydrofolate dehydrogenase (Aldh1/1) were also elevated in the liver of $d b / d b$ mice. In contrast, expression of $P h g d h$ was reduced by $66 \%$ in $d b / d b$ mice, indicating that de novo serine synthesis may also be limited. These results are consistent with genome- 
scale metabolic modeling of human diabetic liver transcriptional data ${ }^{20}$ and highlight potential mechanisms that drive systemic deficiencies in these amino acids.

Circadian and post-prandial variations in amino acids (or glucose) make diagnosis of metabolic defects challenging. We therefore hypothesized that utilization of a "serine-tolerance test" might better gauge SGOC metabolism in animals and identify subjects with elevated serine disposal. Applying dosages used in human clinical trials $(400 \mathrm{mg} / \mathrm{kg})^{21}$, we orally administered serine to fasted C57BL6/J (WT) mice and quantified plasma amino acid pharmacokinetics to gauge the dynamics of serine clearance (Extended Data Fig. 1d). To identify the most critical pathways involved in serine disposal, we next orally administered $\left[\mathrm{U}-{ }^{13} \mathrm{C}_{3}\right]$ serine to overnight fasting WT mice and quantified enrichment of downstream metabolites (Fig. 1c). Remarkably, glucose was labeled at similar levels to glycine throughout the test (Fig. 1d). Indeed, carbon from oral [U${ }^{13} \mathrm{C}_{3}$ ] serine was robustly incorporated into glycine, pyruvate, and citrate within the liver (Fig. 1e-f, Extended Data Fig. 1e-f), demonstrating that Sds-mediated hepatic gluconeogenesis is a major pathway for serine disposal in some contexts and linking its regulation to insulin and glucagon signaling, which are both elevated in $d b / d b$ mice (Fig. 1g-h). To test whether insulin resistanceinduced dysregulation of gluconeogenic flux drives accelerated serine disposal, we delivered both glucose $(2 \mathrm{~g} / \mathrm{kg}$ ) and serine $(400 \mathrm{mg} / \mathrm{kg})$ to fasted WT and $d b / d b$ mice. When comparing serine tolerance in these cohorts, we observed a significant reduction in AUC SER in $d b / d b$ mice, despite administering 2-fold more serine (Fig. 1i-j, Extended Data Fig. 1g). On the other hand, there were no differences in AUC $C_{\text {SER }}$ between WT and $d b / d b$ mice when serine was administered alone (Extended Data Fig. 1h). Interestingly, acute serine challenge had essentially no impact on circulating glycine concentrations (Extended Data Fig. 1i), despite evidence of their rapid interconversion (Fig. 1d-e).

Clinical studies have implicated serine deficiency in the regulation of retinopathy and peripheral neuropathy ${ }^{18,19}$. Given the impaired serine homeostasis in $d b / d b$ mice, we next confirmed that aberrant serine metabolism is coincident with PN. Mice exhibited thermal hypoalgesia, decreased tactile sensation, and decreased motor nerve conduction velocity (MNCV) indicative of PN (Extended Data Fig. 1 $\mathrm{j}-\mathrm{I}$ ). Collectively, these findings suggest that quantifying the dynamics of amino acid metabolism in response to a serine challenge could serve as a diagnostic for patients with a serine-opathy that are susceptible to PN.

\section{Systemic serine deficiency remodels fatty acid metabolism and mitigates adiposity}

Serine and glycine restriction is widely used to modulate health outcomes in mice and is relatively well-tolerated for several months ${ }^{18,22-25}$. To model the impact of systemic serine deficiency in the context of diet-induced obesity, we fed mice low fat ( $10 \%$ kcal, LFD) or high fat ( $60 \%$ fat, HFD) diets and compared their phenotypes to mice fed corresponding, isonitrogenous diets lacking serine and glycine (-SG LFD or -SG HFD; Supplementary Table 1). Both -SG diets effectively reduced circulating and hepatic serine and glycine contents, and HFD alone also reduced liver glycine pools significantly but to a lesser extent than dietary SG withdrawal (Extended Data Fig. $2 a-b)$. Although various hepatic metabolic pools were altered with these dietary interventions, other serine/glycine-derived hepatic metabolites including glutathione were not strongly impacted by their restriction (Extended Data Fig. 2b). Interestingly, weight gain caused by HFD was attenuated by dietary serine/glycine restriction, with -SG HFD mice showing a $\sim 35 \%$ reduction relative to HFD mice (Fig. 2a, Extended Data Fig. 2c). Notably, food, calorie, and water intake, calorie absorption, and physical activity were unaffected (Extended Data Fig. 2d-h). Using echo magnetic resonance imaging (echoMRI), we quantified lean and fat masses across all groups and 
observed that dietary serine restriction had no impact on lean mass, but -SG HFD significantly reduced fat mass relative to the HFD group (Fig. 2b). Consistent with these changes in adiposity, we observed that HFD feeding increased, while serine/glycine restriction diminished, adipocyte size (Extended Data Fig. 2i).

Ectopic visceral adiposity is a hallmark of impaired glucose sensitivity ${ }^{26}$. To determine how -SG HFD feeding influences glucose homeostasis, we subjected mice to a standard glucose tolerance test. While -SG HFD attenuated obesity caused by HFD alone, these mice remained glucose intolerant (Fig. 2c, Extended Data Fig. 2j), suggesting that serine/glycine restriction and the consequent reduction in adiposity do not prevent HFD-induced glucose intolerance. Altered systemic substrate utilization of carbohydrates versus lipids could also drive these serineassociated changes in adiposity. To this end we placed mice that had consumed each diet for 18 weeks in metabolic cages and quantified respiratory exchange ratio (RER). While changing the balance of dietary fat and carbohydrates altered RER as expected, no changes were observed with dietary serine/glycine restriction (Extended Data Fig. 2k).

Gut microbiota can buffer against extreme dietary deficiencies, as evidenced by the strong correlations and empirical associations established between microbiome composition and obesity ${ }^{27,28}$. To understand how the above dietary interventions impacted microbiome composition and diversity, we performed a metagenomic analysis of fecal microbiota and examined their phylogenetic diversities. Sustained HFD reduced alpha diversity relative to LFD fed mice (Fig. $2 \mathrm{~d}$ ), consistent with previous studies ${ }^{29,30}$. Interestingly, while the microbiome of -SG LFD mice exhibited increased phylogenetic alpha diversity, there was no increase in the alpha diversity observed with serine/glycine restriction on the HFD background (Fig. 2d). Next, using robust principal-component analysis (RPCA), we examined how each diet influenced the beta diversity of the microbiome (Fig. 2e). PERMANOVA analysis of RPCA Aitchison distances revealed significant beta-diversity differences between -SG HFD fed mice and other groups $(P=0.0002)$, highlighting the distinct impact of this diet. To gain more functional insights into the metabolic capacity of microbiota across these diets, we calculated the log-ratio of those microbial strains expressing complete pathways of interest given the observed intervention and phenotype: serine biosynthesis, glycine cleavage, and fatty acid biosynthesis. As expected, the log ratio of strains expressing complete serine biosynthesis and glycine cleavage pathways were increased and decreased, respectively, by -SG HFD (Fig. $2 f$ and g). Intriguingly, this intervention also decreased microbial strains with complete fatty acid synthesis pathways (Fig. $2 \mathrm{~h}$ ). On the other hand, serine/glycine restriction on a LFD background did not modulate strains expressing these metabolic pathways, presumably due to the higher carbohydrate content which can be more easily metabolized to generate serine than fat. Key strains showing the strongest alterations are listed in Extended Data Fig. 2l. These results reflect the compensatory mechanisms of the microbiota to restore serine and glycine levels and also link serine/glycine restriction to lipid metabolism.

To directly investigate the impact of serine deficiency on lipid biosynthesis, mice fed the above diets for 18 weeks were administered heavy water $\left(\mathrm{D}_{2} \mathrm{O}\right)$ for $18 \mathrm{~h}$, and lipids were extracted for quantitation of isotope enrichment, molar abundance, and synthesis in liver ${ }^{31}$. In both LFD and HFD backgrounds, dietary serine/glycine restriction potently reduced hepatic palmitate synthesis by $\sim 70 \%$ relative to serine replete control diets (Fig. 2i, Extended Data Fig. $2 \mathrm{~m}$ ). On the other hand, hepatic cholesterol synthesis was increased in -SG HFD compared to HFD (Fig. 2j), indicating distinct regulation of acetyl-CoA pools rather than lipid synthesis in general. Consistent 
with these changes, hepatic expression of ATP-citrate lyase (ACLY), acetyl-CoA carboxylase (ACC2), and stearoyl-CoA desaturase (SCD1) was strongly reduced ( 50\%) by dietary serine restriction (Fig. 2k, Extended Data Fig. 2n-o). In contrast, expression of genes associated with cholesterol biosynthesis was either unchanged or increased in livers from mice fed -SG HFD for 18 weeks (Extended Data Fig. 2p). As noted above, -SG HFD mice still exhibit glucose intolerance (Fig. 1c), and insulin signaling regulates key genes in fatty acid synthesis ${ }^{32}$. We therefore quantified hepatic Akt phosphorylation to gauge insulin signaling changes associated with each diet, observing that $\mathrm{Ser}^{473}$ and $\mathrm{Thr}^{308}$ phosphorylations were significantly reduced by -SG HFD feeding (Fig. 2k, Extended Data Fig. 2n). Collectively, these data suggest that serine/glycine restriction attenuates HFD-induced adiposity gains by reducing hepatic insulin signaling and fatty acid synthesis.

\section{Dyslipidemia accelerates peripheral neuropathy in the context of serine deficiency}

Systemic serine deficiency has recently been linked to various neurodegenerative disorders ${ }^{17,18,33-}$ 35. Diabetics with elevated serine disposal or other genetic predispositions could therefore be most susceptible to neurological co-morbidities reminiscent of putative serine-opathies. To determine if serine deficiency per se was sufficient to drive thermal hypoalgesia, we fed mice with either control or serine/glycine free (-Ser/Gly) diets for up to 12 months. Temporal quantification of thermal response latency to heat revealed no changes after 4 months of feeding with progression to hypoalgesia after 12 months of dietary intervention (Fig. 3a), consistent with prior observations ${ }^{18}$. At this time point we also detected reduced intraepidermal nerve fiber (IENF) density (Fig. 3b, Extended Data Fig. 3a), a clinical measure of PN-associated neurodegeneration ${ }^{36}$. To investigate if serine deficiency accelerates the onset of $P N$ during prolonged lipid overload, we fed mice HFD or -SG HFD for 3 months and quantified thermal latency. Intriguingly, mice fed -SG HFD exhibited marked thermal hypoalgesia, while mice fed a serine/glycine-replete HFD for 3 months showed slight thermal hyperalgesia (Fig. 3c), indicating that the combination of low systemic serine and HFD promote PN in relatively young mice.

Serine is essential for biosynthesis of sphingolipids, which are enriched in the nervous system. Given the importance of ceramides and 1-deoxy(dihydro)ceramides in obesity and some forms of neuropathy ${ }^{37-39}$, we hypothesized that serine palmitoyltransferase (SPT) inhibition could influence these phenotypes. Thus, we administered an inhibitor of SPT, myriocin $(0.3 \mathrm{mg} / \mathrm{kg}$ every other day), or vehicle to mice fed the defined diets described above over 6 months. Consistent with previous reports ${ }^{40,41}$, myriocin treatment attenuated diet-induced weight gain and adiposity relative to HFD vehicle-treated animals without affecting the systemic plasma serine concentration (Fig. 3d, Extended Data Fig. 3b-d). Interestingly, myriocin reduced body weight gain to the same extent as dietary serine restriction and had an additive effect in combination (Fig. 3d, Extended Data Fig. 3b-c). With respect to thermal sensation, myriocin attenuated -SG HFDinduced thermal hypoalgesia (Fig. 3e), suggesting that a reduction in SPT activity mitigates serine-associated PN. Notably, within this cohort neither serine restriction on the LFD background, nor HFD feeding alone, induced thermal hypoalgesia.

To better understand metabolic drivers of this PN phenotype, we next quantified ceramides and 1-deoxysphingolipids in these animals. Interestingly, diet and myriocin had distinct impacts on the ceramide profile in the liver, eWAT, and sciatic nerve (Extended Data Fig. 3e-h). Both -SG LFD and -SG HFD mice exhibited decreased hepatic and adipose canonical ceramides and elevated 1-deoxydihydroceramide and 1-deoxyceramide species, and myriocin generally reduced sphingolipid abundance (Extended Data Fig. 3f-h). On the other hand, ceramide and 1- 
deoxysphingolipid levels were unaltered or did not correlate with PN phenotype in the sciatic nerve (Extended Data Fig. 3f-h). Collectively, these findings demonstrate that inhibition of sphingolipid biosynthesis mitigates development and progression of PN, yet they also highlight that accumulation of 1-deoxysphingolipids alone is insufficient to drive serine-associated thermal hypoalgesia, consistent with recent reports of an SPTLC1 ${ }^{\mathrm{C} 133 \mathrm{~W}}$ mouse model ${ }^{42}$.

\section{SPT inhibition attenuates serine-associated peripheral nerve loss and lipid remodeling}

To gain further mechanistic insights into this diet-induced PN phenotype, we measured IENF density, corneal nerve density, and tissue lipids in a cohort of mice administered LFD, HFD, -SG HFD, or -SG HFD + myriocin for 6 months. As shown above, myriocin delayed the onset and mitigated the severity of thermal hypoalgesia relative to other diets and vehicle treatment (Fig. 4a). Quantification of intraepidermal and corneal nerves revealed that myriocin prevented loss of unmyelinated nerve fibers in response to serine/glycine restriction and lipid overload (Fig. 4b-c, Extended Data Fig. 4a-b). Using high-resolution mass spectrometry, we quantified lipids in the liver and sciatic nerve, focusing on species related to serine and triacylglyceride (TAG) metabolism. Mice fed -SG HFD exhibited increased hepatic 1-deoxysphingolipids, sphingomyelin, and phosphatidylethanolamine (PE)/phosphatidylcholine $(P C)$ ratio but decreased ceramides (Fig. 4d-e, Extended Data Fig. 4c-f). Myriocin potently normalized 1-deoxysphingolipids and sphingomyelin while reducing ceramides and TAGs within the liver (Fig. 4d-e, Extended Data Fig. $4 \mathrm{c}$ and $\mathrm{g}$ ), suggesting that SPT inhibition blunts dispensation of lipids to adipose and other peripheral tissues ${ }^{40}$.

In the sciatic nerve, -SG HFD increased levels of 1-deoxysphingolipids, phosphatidylethanolamine (PE), PE/phosphatidylcholine (PE/PC) ratio, ceramides, and sphingomyelins (Fig. 4f-h, Extended Data Fig. 4i-j, m). Myriocin normalized levels of 1deoxysphingolipids and PE as well as the PE/PC ratio (Fig. 4f-h, Extended Data Fig. 4m-n). On the other hand, sciatic nerve diacylglycerides (DAGs) and TAGs, which may be influenced by the liver ${ }^{43}$, were significantly reduced by myriocin treatment (Fig. 4i, Extended Data 4k). These species have been associated with diabetic $\mathrm{PN}^{44}$, and their reduction in response to myriocin treatment suggests that broader dyslipidemia, rather than insufficient sphingolipid biosynthesis ${ }^{45,46}$, contributes to neuropathy associated with systemic serine deficiency. Indeed, overproduction of sphingolipids can also drive neurological defects ${ }^{45,47}$. Other critical SGOC metabolites such as nucleotides and reduced glutathione (GSH) were not specifically altered in sciatic nerve by -SG HFD (Extended Data Fig. 40). Together, these findings identify key metabolic alterations within the liver and peripheral nervous system involving serine and sphingolipid metabolism that converge to drive PN. In fact, these changes also contribute to onset of small fiber neuropathy in a genetic model of type 2 diabetes, as administration of myriocin to $d b / d b$ mice for 8 weeks mitigated progression to thermal hypoalgesia without impacting body weight gain or onset of hyperglycaemia (Fig. 4j-I), further highlighting the contribution of hepatic sphingolipid metabolism to this neuropathy phenotype.

\section{Discussion}

Here we describe how direct or indirect induction of a "serine-opathy" in mice alters hepatic lipid homeostasis to drive PN. Attenuating aberrant sphingolipid biosynthesis mitigates this phenotype. These results highlight how serine deficiency can synergize with dyslipidemia to alter neurological phenotypes both in rare disease contexts ${ }^{17,18,35}$ and indirectly via a widespread disease like type 2 diabetes, where it manifests as a co-morbidity experienced by a subset of patients (Fig. 4p). A 
serine tolerance test, analogous to an oral glucose tolerance test, could identify patients that exhibit elevated serine/glycine disposal and who might be particularly susceptible to PN. However, these results also suggest that severely diabetic patients might be refractory to serine supplementation, a focus of numerous clinical trials for various neurodegenerative disorders ${ }^{21,38}$.

Hepatic serine/glycine deficiency in the liver of diabetic mice is driven, in part, by increased flux through Sds and gluconeogenesis as well as one carbon metabolism and Gldc. Additional factors impacting serine/glycine availability in these contexts likely include renal metabolism and disposal of acylglycines ${ }^{48}$, which are also influenced by dyslipidaemia ${ }^{49}$. In turn, hepatic sphingolipid biosynthesis and promiscuity manifests these lipid changes throughout the body to impact obesity and accelerate and/or exaggerate neuropathy. Surprisingly, SPT inhibition mitigates -SG phenotypes in unmyelinated nerve fibers of the epidermis and cornea of mice exposed to HFD that are putatively dependent upon canonical sphingolipid availability, potentially through suppression of peripheral doxSL accumulation. At the same time, aberrant lipid accumulation (i.e. $\mathrm{SM}, \mathrm{PE} / \mathrm{PC}$ ) in peripheral nerves further compromises this system to accelerate thermal hypoalgesia, such that "two hits" contribute to accelerated disease onset. While age is a common driver of neuropathy ${ }^{50}$, our results highlight that a combination of metabolic stresses such as serine deficiency and dyslipidaemia can drive PN in relatively young mice. Finally, our analysis of fecal microbiota further highlights the importance of dietary carbohydrate and fat composition in allowing the microbiome and host to negotiate metabolic deficiencies. Diets with a higher glycaemic index promoted more diversity in the microbiome and mitigated serine-associated PN, further suggesting links between microbial diversity and improved health ${ }^{51}$.

Several key questions remain, including why serine/glycine deprivation suppresses hepatic fatty acid synthesis and gene expression in the liver and microbiome. In addition, other sphingolipid species and/or their mis-localization also likely contribute to this neuropathy phenotype $24,45-47,52$. However, our results collectively highlight serine and glycine deficiency and its impact on lipid metabolism as a driver of PN. We have developed and validated a dietary model of serineassociated PN that achieves a phenotype in 3 months. Such serine-opathies can result from diverse genetic changes, including common single nucleotide polymorphisms and rare coding events ${ }^{17,53,54}$, or are induced environmentally by diabetes-induced rewiring of hepatic metabolism. In both driving doxSL accumulation and compromising the liver's (and other tissues') ability to handle nutritional lipid overload, systemic serine deficiency emerges as a modifier of age- and diabetes-associated neuropathies. 


\section{REFERENCES}

1 Feldman, E. L. et al. Diabetic neuropathy. Nature reviews. Disease primers 5, 41, doi:10.1038/s41572-019-0092-1 (2019).

2 Forbes, J. M. \& Cooper, M. E. Mechanisms of diabetic complications. Physiological reviews 93, 137-188, doi:10.1152/physrev.00045.2011 (2013).

3 Newgard, C. B. Interplay between lipids and branched-chain amino acids in development of insulin resistance. Cell Metab 15, 606-614, doi:10.1016/j.cmet.2012.01.024 (2012).

4 Yang, Q., Vijayakumar, A. \& Kahn, B. B. Metabolites as regulators of insulin sensitivity and metabolism. Nature reviews. Molecular cell biology 19, 654-672, doi:10.1038/s41580-018-0044-8 (2018).

5 Spiegelman, B. M. \& Flier, J. S. Obesity and the regulation of energy balance. Cell 104, 531-543 (2001).

6 Zochodne, D. W. Sensory Neurodegeneration in Diabetes: Beyond Glucotoxicity. International review of neurobiology 127, 151-180, doi:10.1016/bs.irn.2016.03.007 (2016).

$7 \quad$ Eid, S. et al. New insights into the mechanisms of diabetic complications: role of lipids and lipid metabolism. Diabetologia 62, 1539-1549, doi:10.1007/s00125-019-4959-1 (2019).

8 Felig, P., Marliss, E. \& Cahill, G. F., Jr. Plasma amino acid levels and insulin secretion in obesity. The New England journal of medicine 281, 811-816, doi:10.1056/nejm196910092811503 (1969). Huffman, K. M. et al. Relationships between circulating metabolic intermediates and insulin action in overweight to obese, inactive men and women. Diabetes care 32, 1678-1683, doi:10.2337/dc08-2075 (2009).

10 Newgard, C. B. et al. A branched-chain amino acid-related metabolic signature that differentiates obese and lean humans and contributes to insulin resistance. Cell Metab 9, 311-326, doi:10.1016/j.cmet.2009.02.002 (2009).

11 Ducker, G. S. et al. Reversal of Cytosolic One-Carbon Flux Compensates for Loss of the Mitochondrial Folate Pathway. Cell Metab 23, 1140-1153, doi:10.1016/j.cmet.2016.04.016 (2016). Parker, S. J. \& Metallo, C. M. Chasing One-Carbon Units to Understand the Role of Serine in Epigenetics. Molecular cell 61, 185-186, doi:10.1016/j.molcel.2016.01.006 (2016).

13 Bervoets, L. et al. Metabolic profiling of type 1 diabetes mellitus in children and adolescents: a case-control study. Diabetology \& metabolic syndrome 9, 48, doi:10.1186/s13098-017-0246-9 (2017).

14 Thalacker-Mercer, A. E. et al. BMI, RQ, diabetes, and sex affect the relationships between amino acids and clamp measures of insulin action in humans. Diabetes 63, 791-800, doi:10.2337/db130396 (2014).

15 Gaggini, M. et al. Altered amino acid concentrations in NAFLD: Impact of obesity and insulin resistance. Hepatology (Baltimore, Md.) 67, 145-158, doi:10.1002/hep.29465 (2018). Laidlaw, S. A. et al. Patterns of fasting plasma amino acid levels in chronic renal insufficiency: results from the feasibility phase of the Modification of Diet in Renal Disease Study. American journal of kidney diseases : the official journal of the National Kidney Foundation 23, 504-513, doi:10.1016/s0272-6386(12)80371-4 (1994).

17 Eade, K. et al. Serine biosynthesis defect due to haploinsufficiency of PHGDH causes retinal disease. Nature metabolism 3, 366-377, doi:10.1038/s42255-021-00361-3 (2021).

18 Gantner, M. L. et al. Serine and Lipid Metabolism in Macular Disease and Peripheral Neuropathy. New England Journal of Medicine, doi:DOI: 10.1056/NEJMoa1815111 (2019).

19 Fridman, V. et al. Altered plasma serine and 1-deoxydihydroceramide profiles are associated with diabetic neuropathy in type 2 diabetes and obesity. Journal of diabetes and its complications $\mathbf{3 5}$, 107852, doi:10.1016/j.jdiacomp.2021.107852 (2021).

20 Mardinoglu, A. et al. Genome-scale metabolic modelling of hepatocytes reveals serine deficiency in patients with non-alcoholic fatty liver disease. Nature communications 5, 3083, doi:10.1038/ncomms4083 (2014).

21 Stark, A. Phase Ila L-serine Trial for eAD (LSPI-2). ClinicalTrials.gov Identifier: NCT03062449 (2017).

22 LeBoeuf, S. E. et al. Activation of Oxidative Stress Response in Cancer Generates a Druggable Dependency on Exogenous Non-essential Amino Acids. Cell Metab 31, 339-350.e334, doi:10.1016/j.cmet.2019.11.012 (2020). 
Maddocks, O. D. et al. Serine starvation induces stress and p53-dependent metabolic remodelling in cancer cells. Nature 493, 542-546, doi:10.1038/nature11743 (2013).

24 Muthusamy, T. et al. Serine restriction alters sphingolipid diversity to constrain tumour growth. Nature, doi:10.1038/s41586-020-2609-x (2020).

25 Gheller, B. J. et al. Extracellular serine and glycine are required for mouse and human skeletal muscle stem and progenitor cell function. Molecular metabolism 43, 101106, doi:10.1016/j.molmet.2020.101106 (2021).

26 Sun, K., Kusminski, C. M. \& Scherer, P. E. Adipose tissue remodeling and obesity. The Journal of clinical investigation 121, 2094-2101, doi:10.1172/jci45887 (2011).

27 Ridaura, V. K. et al. Gut microbiota from twins discordant for obesity modulate metabolism in mice. Science (New York, N.Y.) 341, 1241214, doi:10.1126/science.1241214 (2013). Ley, R. E. et al. Obesity alters gut microbial ecology. Proceedings of the National Academy of Sciences of the United States of America 102, 11070-11075, doi:10.1073/pnas.0504978102 (2005).

29 Turnbaugh, P. J. et al. A core gut microbiome in obese and lean twins. Nature 457, 480-484, doi:10.1038/nature07540 (2009). Walters, W. A., Xu, Z. \& Knight, R. Meta-analyses of human gut microbes associated with obesity and IBD. FEBS letters 588, 4223-4233, doi:10.1016/j.febslet.2014.09.039 (2014). Wallace, M. et al. Enzyme promiscuity drives branched-chain fatty acid synthesis in adipose tissues. Nature chemical biology 14, 1021-1031, doi:10.1038/s41589-018-0132-2 (2018). just a shunting yard for glucose. Biological reviews of the Cambridge Philosophical Society $\mathbf{9 1}$, 452-468, doi:10.1111/brv.12178 (2016). Le Douce, J. et al. Impairment of Glycolysis-Derived I-Serine Production in Astrocytes Contributes to Cognitive Deficits in Alzheimer's Disease. Cell Metab 31, 503-517.e508, doi:10.1016/j.cmet.2020.02.004 (2020).

34 Clemons, T. E. et al. Medical characteristics of patients with macular telangiectasia type 2 (MacTel Type 2) MacTel project report no. 3. Ophthalmic epidemiology 20, 109-113, doi:10.3109/09286586.2013.766757 (2013).

35 Bonelli, R. et al. Systemic lipid dysregulation is a risk factor for macular neurodegenerative disease. Scientific reports 10, 12165, doi:10.1038/s41598-020-69164-y (2020).

36 Kennedy, J. M. \& Zochodne, D. W. Experimental diabetic neuropathy with spontaneous recovery: is there irreparable damage? Diabetes 54, 830-837, doi:10.2337/diabetes.54.3.830 (2005).

37 Chaurasia, B. et al. Targeting a ceramide double bond improves insulin resistance and hepatic steatosis. Science (New York, N.Y.) 365, 386-392, doi:10.1126/science.aav3722 (2019). Fridman, V. et al. Randomized trial of I-serine in patients with hereditary sensory and autonomic neuropathy type 1. Neurology 92, e359-e370, doi:10.1212/wnl.0000000000006811 (2019). Othman, A. et al. Plasma deoxysphingolipids: a novel class of biomarkers for the metabolic syndrome? Diabetologia 55, 421-431, doi:10.1007/s00125-011-2384-1 (2012). Inflammation, and Metabolism. Cell Metab 24, 820-834, doi:10.1016/j.cmet.2016.10.002 (2016). resistance and enhances whole-body oxygen consumption. Diabetes 59, 2453-2464, doi:10.2337/db09-1293 (2010).

42 Hines, T. J. et al. Precision mouse models of Yars/dominant intermediate Charcot-Marie-Tooth disease type $C$ and Sptlc1/hereditary sensory and autonomic neuropathy type 1 . Journal of anatomy, doi:10.1111/joa.13605 (2021).

43 Petersen, M. C. \& Shulman, G. I. Roles of Diacylglycerols and Ceramides in Hepatic Insulin Resistance. Trends in pharmacological sciences 38, 649-665, doi:10.1016/j.tips.2017.04.004 (2017).

44 O'Brien, P. D. et al. Integrated lipidomic and transcriptomic analyses identify altered nerve triglycerides in mouse models of prediabetes and type 2 diabetes. Disease models \& mechanisms 13, doi:10.1242/dmm.042101 (2020).

45 Mohassel, P. et al. Childhood amyotrophic lateral sclerosis caused by excess sphingolipid synthesis. Nature medicine 27, 1197-1204, doi:10.1038/s41591-021-01346-1 (2021). 
Clark, A. J. et al. An iPSC model of hereditary sensory neuropathy-1 reveals L-serine-responsive deficits in neuronal ganglioside composition and axoglial interactions. Cell reports. Medicine 2, 100345, doi:10.1016/j.xcrm.2021.100345 (2021).

47 Clarke, B. A. et al. The Ormdl genes regulate the sphingolipid synthesis pathway to ensure proper myelination and neurologic function in mice. eLife 8, doi:10.7554/eLife.51067 (2019).

48 Leung, K. Y. et al. Regulation of glycine metabolism by the glycine cleavage system and conjugation pathway in mouse models of non-ketotic hyperglycinemia. Journal of inherited metabolic disease 43, 1186-1198, doi:10.1002/jimd.12295 (2020).

49 Floegel, A. et al. Identification of serum metabolites associated with risk of type 2 diabetes using a targeted metabolomic approach. Diabetes 62, 639-648, doi:10.2337/db12-0495 (2013).

$50 \quad$ Brisset, M. \& Nicolas, G. Peripheral neuropathies and aging. Geriatrie et psychologie neuropsychiatrie du vieillissement 16, 409-413, doi:10.1684/pnv.2018.0768 (2018).

51 Tilg, H. \& Kaser, A. Gut microbiome, obesity, and metabolic dysfunction. The Journal of clinical investigation 121, 2126-2132, doi:10.1172/jci58109 (2011).

52 Truman, J. P. et al. Sphingosine kinase 1 downregulation is required for adaptation to serine deprivation. FASEB journal : official publication of the Federation of American Societies for Experimental Biology 35, e21284, doi:10.1096/fj.202001814RR (2021). Scerri, T. S. et al. Genome-wide analyses identify common variants associated with macular telangiectasia type 2. Nature genetics 49, 559-567, doi:10.1038/ng.3799 (2017).

54 Bonelli, R. et al. Identification of genetic factors influencing metabolic dysregulation and retinal support for MacTel, a retinal disorder. Communications biology 4, 274, doi:10.1038/s42003-02101788-w (2021).

Zhang, Y., Huo, M., Zhou, J. \& Xie, S. PKSolver: An add-in program for pharmacokinetic and pharmacodynamic data analysis in Microsoft Excel. Computer methods and programs in biomedicine 99, 306-314, doi:10.1016/j.cmpb.2010.01.007 (2010).

56 Fernandez, C. A., Des Rosiers, C., Previs, S. F., David, F. \& Brunengraber, H. Correction of 13C mass isotopomer distributions for natural stable isotope abundance. Journal of mass spectrometry : JMS 31, 255-262, doi:10.1002/(sici)1096-9888(199603)31:3<255::Aidjms290>3.0.Co;2-3 (1996).

57 Gonzalez, A. et al. Qiita: rapid, web-enabled microbiome meta-analysis. Nature methods 15, 796798, doi:10.1038/s41592-018-0141-9 (2018).

58 Chen, S., Zhou, Y., Chen, Y. \& Gu, J. fastp: an ultra-fast all-in-one FASTQ preprocessor. Bioinformatics (Oxford, England) 34, i884-i890, doi:10.1093/bioinformatics/bty560 (2018).

59 Li, H. Minimap2: pairwise alignment for nucleotide sequences. Bioinformatics (Oxford, England) 34, 3094-3100, doi:10.1093/bioinformatics/bty191 (2018).

60 Langmead, B. \& Salzberg, S. L. Fast gapped-read alignment with Bowtie 2. Nature methods 9 , 357-359, doi:10.1038/nmeth.1923 (2012).

61 Zhu, Q. et al. Phylogenomics of 10,575 genomes reveals evolutionary proximity between domains Bacteria and Archaea. Nature communications 10, 5477, doi:10.1038/s41467-01913443-4 (2019).

62 Bolyen, E. et al. Reproducible, interactive, scalable and extensible microbiome data science using QIIME 2. Nature biotechnology 37, 852-857, doi:10.1038/s41587-019-0209-9 (2019).

63 Faith, D. P. Conservation evaluation and phylogenetic diversity. Biological Conservation, 1-10 (1992).

64 Martino, C. et al. A Novel Sparse Compositional Technique Reveals Microbial Perturbations. mSystems 4, doi:10.1128/mSystems.00016-19 (2019).

65 Anderson, M. J. Permutational Multivariate Analysis of Variance (PERMANOVA). Wiley StatsRef: Statistics Reference Online, 1-15 (2017).

66 Team, S. D. Stan Modeling Language Users Guide and Reference Manual, 2.27. . (2021).

67 Caspi, R. et al. The MetaCyc database of metabolic pathways and enzymes. Nucleic acids research 46, D633-d639, doi:10.1093/nar/gkx935 (2018).

68 Jolivalt, C. G. et al. Peripheral Neuropathy in Mouse Models of Diabetes. Current protocols in mouse biology 6, 223-255, doi:10.1002/cpmo.11 (2016).

69 Cordes, T. \& Metallo, C. M. Quantifying Intermediary Metabolism and Lipogenesis in Cultured Mammalian Cells Using Stable Isotope Tracing and Mass Spectrometry. Methods in molecular biology (Clifton, N.J.) 1978, 219-241, doi:10.1007/978-1-4939-9236-2_14 (2019). 
70 Antoniewicz, M. R., Kelleher, J. K. \& Stephanopoulos, G. Measuring deuterium enrichment of glucose hydrogen atoms by gas chromatography/mass spectrometry. Analytical chemistry $\mathbf{8 3}$, 3211-3216, doi:10.1021/ac200012p (2011).

71 Lim, E. W. et al. Progressive alterations in amino acid and lipid metabolism correlate with peripheral neuropathy in Polg(D257A) mice. Science advances 7, eabj4077, doi:10.1126/sciadv.abj4077 (2021). 


\section{Acknowledgement}

We thank all members of the Metallo Lab for helpful discussions as well as M. Friedlander and E. Holmes for their input on serine-opathies. This work was supported by the NIH (R01CA234245 to C.M.M.; U54CA132379 NIDDK Mouse Metabolic Phenotyping Centers under the MICROMouse Funding Program DK076169 to M.K.H; R01AG065993 to A.C), a Camille and Henry Dreyfus Teacher-Scholar Award (to C.M.M.), the National Science Foundation (NSF) Faculty Early Career Development (CAREER) Program (1454425 to C.M.M.), Lowy Medical Research Institute (to C.M.M), and the American Heart Association (18CDA34110292 to A.C). This work was also supported by the NIDDK-funded San Diego Digestive Diseases Research Center grant (P30 DK120515).

\section{Author contributions}

C.M.M. and M.K.H. designed the study. M.K.H., J.M.G., A.M., Y.I., K.F., and R.J.F. performed animal experiments. M.K.H., G.H.M., C.R.G., generated and analysed targeted metabolomics data. C.M., G.R., and A.G., performed microbiome analysis. T.L. and A.C. performed fecal bomb calorimetry experiments. M.W., P.M., R.K., M.L.G., N.A.C., and C.M.M. guided experimental design and analysis. C.M.M. and M.K.H wrote the manuscript with input from all authors. 


\section{FIGURE LEGENDS}

\section{Figure 1. Sources and sinks of altered serine metabolism in diabetes.}

(a) Levels of glycine, serine and methionine in WT and BKS- $d b / d b$ mice in the liver ( $n=6$ per group).

(b) mRNA expression of enzymes regulating serine, glycine and one-carbon metabolism in WT and BKS- $d b / d b$ mice ( $n=6$ per group).

(c) Schematic of serine and glycine biosynthetic and catabolic pathways. Upregulated pathways are in purple, and downregulated are in blue.

(d) mRNA expression of enzymes regulating serine, glycine and one-carbon metabolism in WT and BKS- $d b / d b$ mice ( $n=6$ per group).

(e) Plasma serine, glucose, glycine and methionine labeling fraction (1-M0) in WT mice following $\left[\mathrm{U}-{ }^{13} \mathrm{C}_{3}\right]$ serine oral gavage ( $\mathrm{n}=4$ per time point).

(f) Tissue glycine labeling fraction in WT mice 15 minutes after $\left[\mathrm{U}^{13} \mathrm{C}_{3}\right]$ serine administration via oral gavage ( $n=4$ per tissues).

(g) Tissue pyruvate labeling fraction in WT mice 15 minutes after $\left[\mathrm{U}-{ }^{13} \mathrm{C}_{3}\right]$ serine administration via oral gavage ( $n=4$ per tissues).

(h) Serum insulin in WT and BKS- $d b / d b$ mice ( $\mathrm{n}=6$ per group).

(i) Serum glucagon in WT and BKS- $d b / d b$ mice ( $n=5-6$ per group).

(j) Combined oral glucose (OGTT) and serine tolerance test (STT) in WT and BKS- $d b / d b$ mice ( $n=6$ per group).

(k) STT area under curve $\left(A_{U} C_{S E R}\right)$ and serine dose in WT and BKS-db/db mice $(\mathrm{n}=6$ per group).

Data are mean \pm standard error of mean (SEM) and were analyzed using an independent t-test (a-b, g-h, j), and two-way ANOVA (i). ${ }^{*} P<0.05,{ }^{* *} P<0.01,{ }^{* * *} P<0.001,{ }^{* * *} P<0.0001$.

Figure 2. Systemic serine deficiency restores glucose homeostasis and mitigates obesity.

(a) Body weight time course in mice fed low fat diet (LFD), serine/glycine free LFD (-SG LFD), high fat diet (HFD), and serine/glycine free HFD (-SG HFD) ( $n=13$ per group).

(b) Lean and fat mass in mice ( $n=10-13$ per group).

(c) Glucose tolerance test area under curve in mice fed serine replete and deplete LFD and HFD diet ( $n=10-13$ per group).

(d) Phylogenetic alpha diversity in mice fed serine replete and deplete LFD and HFD diet $(n=9-10)$.

(e) Robust principal-component analysis of microbiome beta-diversity ( $n=9-10$ per group).

(f) Log-ratio of species with complete vs. incomplete pathways for L-serine biosynthesis $(n=9-10)$.

(g) Log-ratio of species with complete vs. incomplete pathways for glycine cleavage $(n=9-10)$.

(h) Log-ratio of species with complete vs. incomplete pathways for fatty acid synthesis ( $n=9$ 10).

(i) De novo palmitate synthesis in the liver ( $n=3-5$ per group).

(j) De novo cholesterol synthesis in the liver ( $n=3-5$ per group).

(k) Hepatic western blot of liver ACLY, ACC, SCD1, total Akt and phosphorylated Akt protein ( $n=3$ per group). 
Data are mean \pm standard error of mean (SEM) and were analyzed using two-way ANOVA with Fisher's LSD post hoc test (a-d, f-j) and PERMANOVA test $(\mathrm{e}) .{ }^{*} P<0.05,{ }^{* *} P<0.01,{ }^{* * *} P<$ $0.001,{ }^{* * * *} P<0.0001$ vs. LFD group unless otherwise indicated. $\dagger \dagger \dagger P<0.0001$ vs. LFD group.

Figure 3. Inhibition of de novo sphingolipid biosynthesis decelerates kinetics of serineassociated peripheral neuropathy.

(a) Thermal latency time course in mice fed control or serine/glycine free diet for 12 months ( $n=10$ per group).

(b) Quantification of intraepidermal nerve fiber (IENF) density in mice fed control or serine/glycine free diet ( $n=2-3$ per group).

(c) Thermal latency in low fat diet (LFD), high fat diet (HFD) and serine/glycine free HFD (SG HFD) fed mice 3 months after dietary intervention ( $n=7-8$ per group). Thermal latency in this cohort was quantified using Plantar Test Instrument (Ugo Basile).

(d) Body weight time course in mice fed low fat diet (LFD), serine/glycine free LFD (-SG LFD), high fat diet (HFD), and serine/glycine free HFD (-SG HFD) and treated with either vehicle (Veh) or $0.3 \mathrm{mg} / \mathrm{kg}$ myriocin (Myr) ( $\mathrm{n}=9-10$ per group).

(e) Impact of myriocin on the thermal latency 6 months after the treatment ( $n=9-10$ per group).

Data are mean \pm standard error of mean (SEM) and were analyzed using independent t-test (b), two-way ANOVA with Fisher's LSD post hoc test (a,d), and one-way ANOVA with Fisher's LSD post hoc test (e). ${ }^{*} P<0.05,{ }^{* *} P<0.01,{ }^{* * *} P<0.001,{ }^{* * *} P<0.0001$ vs. LFD + Veh unless otherwise indicated.

Figure 4. Inhibition of de novo sphingolipid biosynthesis nerve atrophy and sciatic nerve lipid remodeling.

(a) Thermal latency time course in mice fed LFD, HFD, -SG HFD, and -SG HFD+Myr (n=1112).

(b) Quantification of intraepidermal nerve fiber (IENF) density in mice treated with vehicle or myriocin during dietary interventions ( $n=7-12$ per group).

(c) Quantification of corneal nerves in mice treated with vehicle or myriocin during dietary interventions ( $n=11-12$ per group).

(d) Hepatic 1-deoxydihydroceramide (deoxyDHCer) content ( $n=11-12$ per group).

(e) Hepatic sphingomyelin (SM) content ( $n=11-12$ per group).

(f) Sciatic nerve 1-deoxydihydroceramide (deoxyDHCer) content ( $n=11-12$ per group).

(g) Sciatic nerve phosphatidylethanolamine (PE) content ( $n=11-12$ per group).

(h) Sciatic nerve phosphatidylethanolamine/phosphatidylcholine (PE/PC) ratio ( $n=11-12$ per group).

(i) Sciatic nerve diacylglycerol (DAG) content ( $n=11-12$ per group).

(j) Body weight in 6-week-old BKS- $d b / d b$ mice treated with vehicle or myriocin $(0.3 \mathrm{mg} / \mathrm{kg})$ for 8 weeks ( $n=9-10$ per group).

(k) Blood glucose in 6-week-old BKS- $d b / d b$ mice treated with vehicle or myriocin $(0.3 \mathrm{mg} / \mathrm{kg})$ for 8 weeks ( $n=9-10$ per group).

(I) Thermal latency time course in 6-week-old BKS- $d b / d b$ mice treated with vehicle or myriocin for 8 weeks $(0.3 \mathrm{mg} / \mathrm{kg})(\mathrm{n}=9-10$ per group). 
Data are mean \pm standard error of mean (SEM) and were analyzed using two-way ANOVA with Fisher's LSD post hoc test (a, j-I) and one-way ANOVA with Fisher's LSD post hoc test (b-i). ${ }^{*} P$ $<0.05,{ }^{* *} P<0.01,{ }^{* * *} P<0.001,{ }^{* * *} P<0.0001$. 


\section{EXTENDED DATA FIGURE LEGENDS}

\section{Extended Data Figure 1.}

(a) Levels of glycine, serine and methionine in WT and BKS- $d b / d b$ mice in the kidney $(\mathrm{n}=6$ per group).

(b) Levels of glycine, serine and methionine in WT and BKS- $d b / d b$ mice in the inguinal adipose tissue (iWAT) ( $n=6$ per group).

(c) Plasma metabolite levels in WT and BKS- $d b / d b$ mice ( $n=6$ per group).

(d) Serine tolerance test (STT) in WT mice ( $n=4$ group dosage).

(e) Tissue serine labeling fraction in WT mice 15 minutes after $\left[\mathrm{U}^{13} \mathrm{C}_{3}\right]$ serine administration via oral gavage ( $n=4$ per tissues).

(f) Tissue citrate labeling fraction in WT mice 15 minutes after $\left[\mathrm{U}^{13} \mathrm{C}_{3}\right]$ serine administration via oral gavage ( $n=4$ per tissues).

(g) Plasma glucose pharmacokinetics and area under curve in WT and BKS-db/db mice following OGTT with glucose dosed at $2 \mathrm{~g} / \mathrm{kg}$ ( $\mathrm{n}=6$ per group).

(h) Plasma serine pharmacokinetics and area under curve in WT and BKS- $d b / d b$ mice following STT with serine dosed per body weight $(400 \mathrm{mg} / \mathrm{kg})(\mathrm{n}=6$ per group).

(i) Plasma glycine pharmacokinetics and area under curve in WT and BKS- $d b / d b$ mice following GTT/STT with serine dosed per body weight ( $n=6$ per group).

(j) Thermal latency in WT and BKS- $d b / d b$ mice ( $n=6$ per group)

(k) Tactile sensation in WT and BKS- $d b / d b$ mice ( $n=6$ per group).

(I) Nerve conduction velocity in WT and BKS- $d b / d b$ mice ( $\mathrm{n}=6$ per group).

Data are mean \pm standard error of mean (SEM) and were analyzed using independent t-test (ac, g-l) and two-way ANOVA with Fisher's LSD post hoc test $(\mathrm{g}-\mathrm{i}) .{ }^{*} P<0.05,{ }^{* *} P<0.01,{ }^{* * *} P<$ $0.001,{ }^{* * *} P<0.0001$.

\section{Extended Data Figure 2.}

(a) Plasma amino acid concentration in mice fed low fat diet (LFD) or high fat diet (HFD) replete or deficient in serine and glycine ( $n=5-8$ per group).

(b) Liver metabolite content in mice fed serine/glycine replete and deplete LFD and HFD diet $(n=9-10)$.

(c) Body weight gain 18 weeks after dietary intervention ( $n=13$ per group).

(d) Food intake in response to dietary feeding with a low fat diet (LFD) or high fat diet (HFD) replete or deficient in serine and glycine ( $n=10-13$ per group).

(e) Calorie intake in response to dietary feeding with a low fat diet (LFD) or high fat diet (HFD) replete or deficient in serine and glycine ( $n=10-13$ per group).

(f) Calorie absorption in response to dietary feeding with a low fat diet (LFD) or high fat diet (HFD) replete or deficient in serine and glycine ( $n=10-13$ per group).

(g) Water intake in response to dietary feeding with a low fat diet (LFD) or high fat diet (HFD) replete or deficient in serine and glycine ( $n=10-13$ per group).

(h) Physical activity in response to dietary feeding with a low fat diet (LFD) or high fat diet (HFD) replete or deficient in serine and glycine ( $n=10-13$ per group).

(i) Representative images and quantification of adipocyte size in response to dietary feeding with a low fat diet (LFD) or high fat diet (HFD) replete or deficient in serine and glycine ( $n=10-13$ per group). 
(j) I.P. glucose tolerance test 18 weeks after feeding with a low fat diet (LFD) or high fat diet (HFD) replete or deficient in serine and glycine ( $n=10-13$ per group).

(k) Respiratory exchange ratio time course ( $n=10-13$ per group).

(I) Log fold change of microbiome species ( $n=9-10$ per group).

(m) Plasma heavy water $\left(D_{2} O\right)$ enrichment ( $n=3-5$ mice per group).

(n) Hepatic protein expression of ATP-citrate lyase (ACLY), acetyl-CoA carboxylase (ACC), and steroyl-CoA desaturase 1 (SCD1), P-Akt ${ }^{\mathrm{S} 473}$, and $\mathrm{P}-\mathrm{Akt}^{\mathrm{T} 308}$ ( $\mathrm{n}=3$ per group).

(o) Hepatic mRNA expression of ATP-citrate lyase (Acly), acetyl-CoA carboxylase (Acc2), and steroyl-CoA desaturase 1 (Scd1) ( $n=8-13$ per group).

(p) Hepatic mRNA expression of enzymes involved in fatty acid and cholesterol synthesis ( $n=8-13$ per group).

Data are mean \pm standard error of mean (SEM) and were analyzed using two-way ANOVA with Fisher's LSD post hoc test (a-j, m-p). ${ }^{*} P<0.05,{ }^{* *} P<0.01,{ }^{* * *} P<0.001,{ }^{* * * *} P<0.0001$ vs. LFD unless otherwise indicated. †† $P<0.01$ vs. LFD. \# $P<0.05$ vs. LFD.

\section{Extended Data Figure 3.}

(a) Representative images of intraepidermal nerve fiber (IENF) density in mice fed control or serine/glycine free diet. PGP9.5 staining of IENF is shown by arrows.

(b) Body weight gain 6 months after dietary intervention and myriocin treatment $(n=9-10$ per group).

(c) Adipose tissue size 6 months after dietary intervention and myriocin treatment $(n=9-10$ per group).

(d) Plasma serine concentration 6 months after dietary intervention and myriocin treatment ( $n=9-10$ per group).

(e) Schematic of serine palmitoyltransferase (SPT) promiscuity and de novo sphingolipid biosynthesis pathway

(f) Liver, epididymal adipose tissue (eWAT), and sciatic nerve ceramide content 6 months after dietary intervention and myriocin treatment ( $n=9-10$ per group).

(g) Liver, epididymal adipose tissue (eWAT), and sciatic nerve deoxydihydroceramide content 6 months after dietary intervention and myriocin treatment ( $n=9-10$ per group).

(h) Liver, epididymal adipose tissue (eWAT), and sciatic nerve deoxyceramide content 6 months after dietary intervention and myriocin treatment ( $n=9-10$ per group).

Data are mean \pm standard error of mean (SEM) and were analyzed using one-way ANOVA with Fisher's LSD post hoc test (b-d, f-h). ${ }^{*} P<0.05,{ }^{* *} P<0.01,{ }^{* * *} P<0.001,{ }^{* * * *} P<0.0001$ vs. LFD + Veh group unless otherwise indicated.

\section{Extended Data Figure 4.}

(a) Representative images of intraepidermal nerve fiber (IENF) density in mice treated with vehicle or myriocin during dietary interventions. PGP9.5 staining of IENF is shown by arrows.

(b) Representative images of corneal nerves in mice treated with vehicle or myriocin during dietary interventions.

(c) Hepatic ceramide (Cer) content ( $n=11-12$ per group).

(d) Hepatic phosphatidylethanolamine (PE) content ( $n=11-12$ per group). 
(e) Hepatic phosphatidylethanolamine (PE)/phosphatidylcholine (PC) ratio $(n=11-12$ per group).

(f) Hepatic phosphatidylcholine (PC) content ( $n=11-12$ per group).

(g) Hepatic triacylglyceride (TAG) content ( $n=11-12$ per group).

(h) Hepatic diacylglyceride (DAG) content ( $n=11-12$ per group).

(i) Sciatic nerve ceramide (Cer) content ( $n=11-12$ per group).

(j) Sciatic nerve sphingomyelin (SM) content ( $n=11-12$ per group).

(k) Sciatic nerve triacylglyceride (TAG) content ( $n=11-12$ per group).

(l) Sciatic nerve phosphatidylcholine (PC) content ( $n=11-12$ per group).

(m) Sciatic nerve distribution of phosphatidylethanolamine (PE) species ( $n=11-12$ per group).

(n) Metabolic map linking serine/glycine metabolism with complex lipids.

(o) Sciatic nerve polar metabolite heatmap ( $n=11-12$ per group).

(p) Schematic illustrating impacts of metabolic syndrome and serine-opathy on serine metabolism and peripheral neuropathy.

Data are mean \pm standard error of mean (SEM) and were analyzed using one-way ANOVA with Fisher's LSD post hoc test (c-l, o), and two-way ANOVA with Fisher's LSD post hoc test (m). ${ }^{*} P$ $<0.05,{ }^{* *} P<0.01,{ }^{* * *} P<0.001$ vs. LFD + Veh group unless otherwise indicated. $\# P<0.05$ vs. LFD + Veh. 


\section{METHODS}

\section{Animal experiments}

All animal experiments were approved and conducted in accordance with the Institutional Animal Care and Use Committee (IACUC) of the University of California San Diego. Fourteen- to sixteenweek-old C57BL/6J (JAX\# 000664) or BKS-db/db mice (JAX \#000642) were fasted for 6 hours prior to tissue collection. Animals were anesthetized with isoflurane and tissues rapidly collected using Wollenberger clamps pre-cooled to the temperature of liquid nitrogen and stored at $-80{ }^{\circ} \mathrm{C}$ until analysis. Dietary experiments were performed with 8-week-old C57BL/6J mice (Jackson Laboratories) with diets obtained from Envigo. Dietary composition is detailed in the Supplementary Table 1. In dietary experiments, tissues were collected between 07.00-10.00am, i.e. in the fed state. In $d b / d b$ mice experiments, tissues were collected after 6-hour fasting.

\section{Serine tolerance test (STT)}

Age-matched fourteen- to sixteen-week-old WT and BKS- $d b / d b$ mice were fasted overnight with water access provided ad libitum. For a STT animals were weighed, and serine and glucose administered via oral gavage at a dose of $400 \mathrm{mg} / \mathrm{kg}$ and $2 \mathrm{~g} / \mathrm{kg}$, respectively, with tail tip blood samples collected into EDTA-coated microvette tubes (Sarstedt Inc.) before, and 15, 30, 60, 120 and 180 minutes after oral gavage. EDTA microvettes were spun at $2000 \mathrm{~g}$ at $4{ }^{\circ} \mathrm{C}$ for 5 minutes to obtain plasma, and samples stored at $-80{ }^{\circ} \mathrm{C}$ until analysis. Blood glucose and serine concentrations were quantified using Contour Next glucometer (Bayer) and gas chromatography mass spectrometry as described below, respectively. Plasma serine pharmacokinetics were determined for $400 \mathrm{mg} / \mathrm{kg}$ dose using PK solver ${ }^{55}$.

To qualify downstream fate of serine, WT mice were fasted overnight, weighed in the morning, and $\left[\mathrm{U}-{ }^{13} \mathrm{C}_{3}\right]$-serine administered via oral gavage at a dose of $400 \mathrm{mg} / \mathrm{kg}$, with tissues collected, using Wollenberger clamps pre-cooled to the temperature of liquid nitrogen, before, and 15, 30, 45,60 , and 120 minutes after oral gavage, and samples stored at $-80{ }^{\circ} \mathrm{C}$ until analysis.

\section{Serum insulin and glucagon}

Commercially available kits were used to determine serum insulin (Mouse Insulin ELISA \#101247-01, Mercodia, Sweden) and glucagon (Glucagon ELISA \#10-1271-01, Mercodia, Sweden) following a 6-hour fasting WT and BKS- $d b / d b$ mice according to the manufacturer's instructions.

\section{$\mathrm{D}_{2} \mathrm{O}$ experiments}

C57BL/6J mice fed diets for 18 weeks were i.p injected with $\mathrm{D}_{2} \mathrm{O}$ (in $0.9 \% \mathrm{NaCl}$ ) at a dose of $0.027 \mathrm{~mL} / \mathrm{g}$ of body weight with drinking water replaced by $6 \% \mathrm{D} 2 \mathrm{O}$ enriched solution for a period of $\sim 18$ hours. In the morning (07.00-10.00am) tissues were rapidly collected using Wollenberger clamps pre-cooled to the temperature of liquid nitrogen and stored at $-80 \mathrm{C}$ until analysis.

Plasma $\mathrm{D}_{2} \mathrm{O}$ enrichment was determined using deuterium-acetone exchange protocol as previously described ${ }^{31}$. Briefly, $5 \mu \mathrm{l}$ of plasma were incubated with $4 \mu \mathrm{l}$ of $5 \%$ acetone in acetonitrile solution and $4 \mu \mathrm{l}$ of $10 \mathrm{M} \mathrm{NaOH}$ for 24 hours. Next, $500 \mathrm{mg}$ of $\mathrm{Na}_{2} \mathrm{SO}_{4}$ and $600 \mu \mathrm{l}$ of chloroform were added, and samples vortex mixed. After 2 min centrifugation at $3000 \mathrm{~g}, 80 \mu \mathrm{l}$ was transferred in triplicate in to a GC-MS vial, and plasma $\mathrm{D}_{2} \mathrm{O}$ enrichment quantified from external standard curve on a Agilent DB-35MS column $(30 \mathrm{~m} 30.25 \mathrm{~mm}$ i.d. $\times 0.25 \mu \mathrm{m}$, Agilent J\&W Scientific) installed in an Agilent 7890 A gas chromatograph (GC) interfaced with an Agilent $5975 \mathrm{C}$ mass spectrometer (MS) with the following temperature program: $60^{\circ} \mathrm{C}$ initial, increase by $20^{\circ} \mathrm{C} / \mathrm{min}$ to $100^{\circ} \mathrm{C}$, increase by $50^{\circ} \mathrm{C} / \mathrm{min}$ to $220^{\circ} \mathrm{C}$, and hold for $1 \mathrm{~min}$. 
To quantify tissue $\mathrm{D}_{2} \mathrm{O}$ labeling, $\sim 20 \mathrm{mg}$ of frozen tissue was homogenized with $250 \mu \mathrm{L}-20{ }^{\circ} \mathrm{C}$ methanol, $250 \mu \mathrm{L}$ ice-cold saline and $500 \mu \mathrm{l}$ of $-20^{\circ} \mathrm{C}$ chloroform spiked with internal standard palmitate-d31 (Cambridge Isotope Laboratories) and coprostanol (Sigma, Cat\# 7578). After a 5 min spin at $4{ }^{\circ} \mathrm{C}$ for $21000 \mathrm{~g}$, chloroform fraction was collected, dried and resuspended with 500 $\mu \mathrm{l}$ of $2 \% 2 \% \mathrm{H}_{2} \mathrm{SO}_{4}$ in methanol for 2 hours at $50{ }^{\circ} \mathrm{C}$. Next, $100 \mu \mathrm{l}$ of saturated $\mathrm{NaCl}$ and $500 \mu \mathrm{l}$ of hexane were added, samples vortex mixed, and upper hexane phase collected and transferred into a GC-MS vial. FAMES were analyzed using a Select FAME column $(100 \mathrm{~m} \times 0.25 \mathrm{~mm}$ i.d. $)$ installed in an Agilent 7890 A GC interfaced with an Agilent $5975 \mathrm{C}$ MS using the following temperature program: $80^{\circ} \mathrm{C}$ initial, increase by $20^{\circ} \mathrm{C} / \mathrm{min}$ to $170{ }^{\circ} \mathrm{C}$, increase by $1{ }^{\circ} \mathrm{C} / \mathrm{min}$ to $204^{\circ} \mathrm{C}$, then $20^{\circ} \mathrm{C} / \mathrm{min}$ to $250^{\circ} \mathrm{C}$ and hold for $10 \mathrm{~min}$. The percent isotopologue distribution of each fatty acid and polar metabolite was determined and corrected for natural abundance using in-house algorithms adapted from a previous report ${ }^{56}$.

\section{Glucose tolerance test}

C57BL/6J mice fed diets for 18 weeks were fasted overnight with water provided ad libitum. In the morning animals were weighed and fasting blood glucose determined from the tail bleed using Contour Next glucometer (Bayer). Next, the animals were i.p. injected with a bolus of glucose at a dose of $2 \mathrm{~g} / \mathrm{kg}$ of body weight, and blood glucose determined at 15, 30, 60, 120 and 180 minutes post-injection.

\section{Determination of body composition and systemic metabolic rates}

Lean and fat masses were determined using EchoMRI 3-in-1 instrument (quantitative nuclear magnetic resonance (qNMR) imaging system). Comprehensive Laboratory Animal Monitoring System (CLAMS) (Oxymax®, Columbus Instruments, Columbus, OH) was used to quantify systemic metabolic rates in individually housed mice during the period of 6 days. Water, food, and calorie intake were calculated from individually housed animals over a period of 6 days when subjected to CLAMS. Whole-body oxygen consumption $\left(\mathrm{VO}_{2}\right)$ and carbon dioxide $\left(\mathrm{VCO}_{2}\right)$ rates were normalized to corresponding total body weights, and respiratory exchange ratio (RER) was calculated as ratio of $\mathrm{VCO}_{2}$ to $\mathrm{VO}_{2}$.

\section{Fecal bomb calorimetry and calorie absorption}

Approximately one gram of feces was desiccated overnight and ground using mortar and pestle. Powdered sample was reconstituted into a pellet with $300 \mu \mathrm{lddH} 2 \mathrm{O}$ and weighed. Pellet was placed in bomb cylinder surrounded by $2000 \mathrm{ml}$ ddH2O (Parr 6100 Compensated Jacket Calorimeter). Heat produced by combustion was measured by change in water temperature. The calorimeter energy equivalent, $\mathrm{W}\left(\mathrm{Cal} /{ }^{\circ} \mathrm{C}\right)$, was calculated with standardized benzoic acid. The final energy content of each pellet was calculated as follows:

$$
\text { Gross energy }(\mathrm{Cal} / \mathrm{g})=\frac{\left(\mathrm{T}_{\text {final }}-\mathrm{T}_{\text {initial }}\right)(\mathrm{W})}{\text { sample weight }(\mathrm{g})}
$$

Calorie absorption was calculated by subtracting gross energy (fecal calorie extraction) from calorie intake.

\section{Microbiome analysis}

DNA was extracted from 10-30 mgs of stool using the MoBio PowerFecal DNA isolation kit (Cat\# 12830-50). Extracted DNA was quantified using a Nanodrop (ThermoFisher Scientific). The whole genome sequencing raw data was uploaded to Qiita ${ }^{57}$, where we followed their default processing workflow. In summary, the raw reads were adaptor filtered using the auto-detect parameters in fastp version $20^{58}$ and host (mouse) filtered using minimap2 version $2.17^{59}$. The resulting 
sequences were aligned using Bowtie 2 version 2.4.260 to the Web of Life (WoL) reference database $^{61}$ via the Web of Life Toolkit App (https://github.com/qiyunzhu/woltka); this step generated tables at genus, species, per genome, and per gene tables. For all analyzes we used the per genome table; then for alpha diversity we removed any samples below 1,273,062 sequences per sample and for beta diversity analysis we rarefied at the same value. Downstream analyses were performed in QIIME 2 version 2020.1162. To asses global microbiota alterations, alpha diversity analysis was performed through Faith's $\mathrm{PD}^{63}$ and beta diversity through robust principal-component analysis (RPCA) ${ }^{64}$ and resulting Aitchison distances were evaluated through permutational multivariate analysis of variance (PERMANOVA) ${ }^{65}$.

We then designed a Bayesian hierarchical model for differential abundance incorporating diet type as a fixed effect and cage as a random effect. We model the count generating process as a negative binomial distribution to account for overdispersion. Due to the sparsity of microbiome data, we also accounted for zero-inflation by assigning each microbe a probability of being unobserved separately from the count generating process:

$\begin{aligned} y_{i j} & = \begin{cases}0, & \theta_{j}=1 \\ \text { NegativeBinomial }\left(\eta_{i j}, \phi_{j}\right), & \theta_{j}=0\end{cases} \\ \theta_{j} & =\operatorname{Bernoulli}\left(\pi_{j}\right)\end{aligned}$

$$
\log \left(\eta_{i j}\right)=x_{i} \beta_{j}+z_{i} u_{j}+\log \left(\operatorname{Depth}_{i}\right)
$$

We wrote this model using the Stan probabilistic programming language ${ }^{66}$ and fit the model using BIRDMAn (https://github.com/gibsramen/BIRDMAn). To account for compositionality, we fit this model using the first microbe in the table as an additive log-ratio reference and converted log-fold changes into centered log-ratio coordinates after fitting. We used the following as prior distributions for the target parameters:

$$
\begin{aligned}
\pi_{j} & \sim \operatorname{Beta}(1.5,1.5) \\
\phi_{j}^{-1} & \sim \operatorname{Cauchy}_{+}(0,3) \\
\beta_{j} & \sim \operatorname{Normal}(0,5) \\
u_{j} & \sim \operatorname{Normal}(0,2)
\end{aligned}
$$

In order to compare functional changes associated with strain level differential abundances a comparative genomics pathway completeness approach was taken. First, each genome was assessed via MetaCyc ${ }^{67}$ pathway completeness, a proportion ranging from zero to one, by mapping characterized genes to reactions and finally to pathways. Each pathway was then correlated by Spearman's rank correlation to the beta differential abundance determined from the above model. Serine biosynthesis, glycine cleavage, and fatty acid synthesis pathways were significantly correlated to betas. To validate these correlations, the log-ratio of the sum of the abundance of genomes with complete pathways (completeness $=1$ ) vs. those without (completeness < 1) were evaluated between treatment groups.

\section{Behavioral assays}

Thermal sensation

Small sensory $\mathrm{C}$ fiber function was quantified by behavioral responses to heat using thermal nociception test device (UARD) as previously described ${ }^{68}$. Briefly, the apparatus surface was 
warmed up to $30{ }^{\circ} \mathrm{C}$, and animals were placed in individual testing chambers for $20-30$ minutes prior to testing. Four separate response latency measurements were performed, and the mean of the last triplicate taken to represent response latency for each animal. In the 3 month cohort (LFD, HFD, -SG HFD), thermal latency was determined using Plantar Test Instrument (Ugo Basile, US).

\section{Tactile sensation}

Animals were placed on the von Frey stand and allowed to acclimate for 20-30 minutes. The range of manual von Frey filaments was used: 2.44, 2.83, 3.22, 3.61, 3.84, 4.08, 4.31, 4.56, 4.74 (Kom Kare, Inc.). Testing began with the 3.84 filament and the pressure applied was repeated five times. If a positive response was observed, the next lower weighted filament was used in the sequence. In the case of a negative response, the next higher weighted filament was applied.

\section{Nerve conduction velocity}

Conduction of a motor nerve was quantified in anesthetized mice using EZ Anesthesia Versaflex system (Braintree Scientific, Cat\# Z-7150). Briefly, lightly anesthetized mice were transferred onto a water-heated pad with anesthesia maintained via a face mask. Two recording platinum electrodes were inserted between the animal's second, third, and fourth toes, and a grounding electrode into the skin at the neck. PowerLab stimulator delivered a 200-mV, 50- $\mu$ sec-duration square-wave stimulus every 2 seconds. The stimulating electrode was inserted in the ankle near the Achilles tendon and subsequently into the sciatic notch at the hip, and $\mathrm{M}$ and $\mathrm{H}$ waves recorded. The latency between Achilles tendon and sciatic notch was used to calculate nerve conduction velocity as described ${ }^{68}$.

\section{Corneal confocal imaging}

Quantification of corneal nerves was performed in anesthetized mice (using EZ Anesthesia Versaflex system, Braintree Scientific, Cat\# Z-7150) using Retina Tomograph 3 with Rostock Cornea Module (Heidelberg Engineering Inc.) equipped with Tomocap (Heidelberg Engineering, cat. no. 0220-001) as previously described ${ }^{68}$. Briefly, lightly anesthetized mice were transferred onto a small animal platform with anesthesia maintained via a face mask. Forty sequential images of uniform magnification and size were collected and those containing nerves of the sub-basal plexus identified. ImageJ software was used to quantify corneal nerve area within each image, with data presented as pixels/image.

\section{Epidermal innervation}

Quantification of epidermal innervation was performed in paw skin samples by immunostaining for the pan-neuronal protein PGP9.5, as described previously in detail ${ }^{68}$. Briefly, paw skin samples were collected into 4\% buffered paraformaldehyde (Thermo scientific, \#J19943-K2). Staining of epidermal nerves was performed using anti-PGP9.5 antibody (supplier, antibody dilution). Using 40x magnification of a light microscope, the number of PGP9.5+ve profiles present in the epidermis was calculated, length of skin section calculated and IENF profile density expressed as profiles $/ \mathrm{mm}$.

\section{Metabolite extraction and quantification}

\section{Metabolite extraction}

Plasma polar metabolites were extracted from $3 \mu \mathrm{L}$ of plasma spiked with a known amount of ${ }^{13} \mathrm{C}$ and ${ }^{15} \mathrm{~N}$ labeled standards (Cat\# MSK-A2-1.2, Cambridge Isotope Laboratories). Tissue metabolite extraction was performed as described before ${ }^{24}$. Briefly, $\sim 20 \mathrm{mg}$ of tissue was homogenized for 2 min using Precellys beads with $500 \mu \mathrm{L}$ of $-20^{\circ} \mathrm{C}$ methanol, $400 \mu \mathrm{L}$ of ice-cold saline and $100 \mu \mathrm{L}$ of ice-cold water and spiked with ${ }^{13} \mathrm{C} /{ }^{15} \mathrm{~N}$ polar metabolite standards (Cat\# 
MSK-A2-1.2, Cambridge Isotope Laboratories), 20 pmol of sphinganine-d7 (Avanti Polar Lipids, Cat\# 860658), 2 pmol of deoxysphinganine-d3 (Avanti Polar Lipids, Cat\# 860474), 100 pmol of 13C-dihydroceramide-d7 (Avanti Polar Lipids, Cat\# 330726), 200 pmol of C15-ceramide-d7 (Avanti Polar Lipids, Cat\# 860681), 10 pmol of d18:1-d7 glucosylsphingosine (Avanti Polar Lipids, Cat\# 860695), 100 pmol of d18:1-d7/15:0 glucosylceramide (Avanti Polar Lipids, Cat\# 330729), 100 pmol of d18:1-d7/15:0 lactosylceramide (Avanti Polar Lipids, Cat\# 330727), 200 pmol of sphingosine-d7 (Avanti Polar Lipids, Cat\# 860657), and 200 pmol of d18:1/18:1-d9 sphingomyelin (Avanti Polar Lipids, Cat\# 791649). Homogenate aliquot of $50 \mu \mathrm{l}$ was taken to determine tissue protein content using BCA protein assay (Lambda Biotech, Cat\# G1002). The remaining homogenate was transferred to a $2 \mathrm{ml}$ Eppendorf tube and $1 \mathrm{ml}$ of $-20{ }^{\circ} \mathrm{C}$ chloroform was added. Samples were vortex mixed for $5 \mathrm{~min}$ and spun down for $5 \mathrm{~min}$ at $4{ }^{\circ} \mathrm{C}$ at $15000 \mathrm{~g}$. The organic phase was collected and $2 \mu \mathrm{l}$ of formic acid was added to the remaining polar phase which was re-extracted with $1 \mathrm{ml}$ of $-20{ }^{\circ} \mathrm{C}$ chloroform. Combined organic phases were dried and the pellet was resuspended in $100 \mu \mathrm{l}$ of buffer containing 100\% methanol, $1 \mathrm{mM}$ ammonium formate and $0.2 \%$ formic acid.

\section{Quantification (gas chromatography mass spectrometry)}

Quantification of polar metabolites was determined after derivatization with $2 \%$ (w/v) methoxyamine hydrochloride (Thermo Scientific) in pyridine $\left(37^{\circ} \mathrm{C}\right.$ for $60 \mathrm{~min}$ ) and with $\mathrm{N}$ tertbutyldimethylsilyl-N-methyltrifluoroacetamide (MTBSTFA) with $1 \%$ tertbutyldimethylchlorosilane (tBDMS) (Regis Technologies) $\left(37^{\circ} \mathrm{C}\right.$ for 30$)$. Polar derivatives were analyzed by GC-MS using a DB-35MS column $(30 \mathrm{~m} \times 0.25 \mathrm{~mm}$ i.d. $\times 0.25 \mu \mathrm{m}$, Agilent J\&W Scientific) installed in an Agilent 7890 A gas chromatograph (GC) interfaced with an Agilent 5975 C mass spectrometer (MS) as previously described ${ }^{69}$. Plasma glucose enrichment was determined using propionic anhydride derivatization as previously described ${ }^{70}$. Natural isotope abundance was corrected using in-house script ${ }^{56}$.

\section{Quantification (high resolution mass spectrometry)}

Around $10 \sim 20 \mathrm{mg}$ of frozen tissue was extracted with $800 \mu \mathrm{l}$ of $-20^{\circ} \mathrm{C}$ 5:3:2 acetonitrile: methanol:water solution spike with a known concentration of norvaline as an internal standard using the Precellys Evolution Homogenizer (Bertin Technologies Inc.) ${ }^{71}$. After extraction, a $50 \mu \mathrm{l}$ aliquot was taken for protein quantification using BCA protein assay (Lambda Biotech, Cat\# $\mathrm{G} 1002$ ), and the remaining extract was spun for $10 \mathrm{~min}$ at $21,000 \mathrm{~g}$ at $4^{\circ} \mathrm{C}$. The supernatant was then transferred into a glass vial, and chromatographic separation and compound identification performed using Q Exactive Orbitrap MS with a Vanquish Flex Binary UHPLC system (Thermo Fisher Scientific) was used with an iHILIC-(P) Classic, $150 \mathrm{~mm}$ by $2.1 \mathrm{~mm}, 5-\mathrm{mm}$ particle, 200- $\AA$ (Hilicon) column at $45^{\circ} \mathrm{C}$. Chromatography was performed using a gradient of $20 \mathrm{mM}$ ammonium carbonate, adjusted to $\mathrm{pH} 9.4$ with $0.1 \%$ ammonium hydroxide $(25 \%)$ solution (mobile phase $\mathrm{A}$ ) and $100 \%$ acetonitrile (mobile phase B), both at a flow rate of $0.2 \mathrm{ml} / \mathrm{min}$. The liquid chromatography (LC) gradient ran linearly from 80 to $20 \%$ B from 2 to 17 min and then from 20 to $80 \% \mathrm{~B}$ from 17 to $18 \mathrm{~min}$ and then held at $80 \% \mathrm{~B}$ from 18 to $25 \mathrm{~min}$.

\section{Targeted sphingolipid quantification}

Quantification of sphingolipid metabolites was determined using triple quadrupole liquid chromatography mass spectrometry platform (6460, Agilent). Sphingolipid species were separated on a C8 column (Spectra $3 \mu \mathrm{m}$ C8SR $150 \times 3 \mathrm{~mm}$ inner diameter, Peeke Scientific) as previously described ${ }^{20}$. Mobile phase A was composed of $100 \%$ HPLC-grade water containing 2 $\mathrm{mM}$ ammonium formate and $0.2 \%$ formic acid and mobile phase B consisted of $100 \%$ methanol containing $0.2 \%$ formic acid and $1 \mathrm{mM}$ ammonium formate. The gradient elution program consisted of the following profile: $0 \mathrm{~min}, 82 \% \mathrm{~B} ; 3 \mathrm{~min}, 82 \% \mathrm{~B} ; 4 \mathrm{~min}, 90 \% \mathrm{~B}, 18 \mathrm{~min}, 99 \% \mathrm{~B} ; 25$ 
$\min , 99 \%, 27 \mathrm{~min}, 82 \% \mathrm{~B}, 30 \mathrm{~min}, 82 \% \mathrm{~B}$. Column re-equilibration followed each sample and lasted $10 \mathrm{~min}$. The capillary voltage was set to $3.5 \mathrm{kV}$, the drying gas temperature was $350^{\circ} \mathrm{C}$, the drying gas flow rate was $10 \mathrm{l} / \mathrm{min}$ and the nebulizer pressure was $60 \mathrm{psi}$. Sphingolipid species were analyzed by selective reaction monitoring (SRM) of the transition from precursor to product ions at associated optimized collision energies and fragmentor voltages (Supplementary Table 2). Quantification of sphingolipid species was performed using spiked in deuterated standards.

\section{Hepatic and sciatic nerve lipidomics}

Liver and sciatic nerves were extracted in $400 \mu \mathrm{l}$ of $-20{ }^{\circ} \mathrm{C}$ methanol using the Precellys Evolution Homogenizer (Bertin Technologies Inc.) spiked with EquiSPLASH labeled standard (Avanti Polar Lipids, Cat\# 330731) and norvaline. After extraction, $50 \mu \mathrm{l}$ aliquot was taken to quantify protein content using BCA protein assay (Lambda Biotech, Cat\# G1002), and to the remaining extract were added $400 \mu \mathrm{l}$ of $-20{ }^{\circ} \mathrm{C}$ chloroform and $400 \mu \mathrm{l}$ of ice-cold water. After vortexing for 5 mins, samples were spun for $5 \mathrm{~min}$ at $4{ }^{\circ} \mathrm{C}$ at $15000 \mathrm{~g}$, and the organic phase was collected. Two $\mu \mathrm{l}$ of formic acid were added to the remaining polar phase which was re-extracted with $400 \mu$ of -20 ${ }^{\circ} \mathrm{C}$ chloroform, samples were vortex and spun and described above. Combined organic phases were dried and the pellet was resuspended in $100 \mu \mathrm{l}$ isopropanol.

Chromatographic separation and lipid species identification was performed using $Q$ Exactive orbitrap mass spectrometer with a Vanquish Flex Binary UHPLC system (Thermo Scientific) equipped with an Accucore C $30,150 \times 2.1 \mathrm{~mm}, 2.6 \mu \mathrm{m}$ particle (Thermo) column at $40^{\circ} \mathrm{C} .5 \mu \mathrm{L}$ of sample was injected. Chromatography was performed using a gradient of 40:60 v/v water: acetonitrile with $10 \mathrm{mM}$ ammonium formate and $0.1 \%$ formic acid (mobile phase $A$ ) and 10:90 v/v acetonitrile: 2-propanol with $10 \mathrm{mM}$ ammonium formate and $0.1 \%$ formic acid (mobile phase B), both at a flow rate of $0.2 \mathrm{~mL} / \mathrm{min}$. The LC gradient ran from $30 \%$ to $43 \% \mathrm{~B}$ from $3-8 \mathrm{~min}$, then from $43 \%$ to $50 \%$ B from $8-9$ min, then $50-90 \%$ B from $9-18 \mathrm{~min}$, then $90-99 \%$ B from $18-26$ min, then held at $99 \%$ B from $26-30 \mathrm{~min}$, before returning to $30 \% \mathrm{~B}$ in $6 \mathrm{~min}$ and held for a further $4 \mathrm{~min}$.

Lipids were analyzed in positive mode using spray voltage $3.2 \mathrm{kV}$. Sweep gas flow was 1 arbitrary units, auxiliary gas flow 2 arbitrary units and sheath gas flow 40 arbitrary units, with a capillary temperature of $325^{\circ} \mathrm{C}$. Full MS (scan range $200-2000 \mathrm{~m} / \mathrm{z}$ ) was used at 70,000 resolution with $1 \mathrm{e} 6$ automatic gain control and a maximum injection time of $100 \mathrm{~ms}$. Data dependent MS2 (Top 6 ) mode at 17,500 resolution with automatic gain control set at 1 e5 with a maximum injection time of $50 \mathrm{~ms}$ was used. Data was analyzed using El-Maven software, and peaks normalized to Avanti EquiSPLASH internal standard. Lipid species specific fragments used for identification and quantitation are presented in the Supplementary Table 3.

\section{Gene expression analysis}

RNA was extracted from $\sim 20$ mg of liver tissue using Direct-Zol RNA kit (Direct-Zol RNA Miniprep Plus kit, Zymo Research) according to the manufacturer's instructions. Brown adipose tissue RNA was extracted from $\sim 20 \mathrm{mg}$ of tissue according to the manufacturer's instructions using RNeasy Lipid Tissue Mini Kit (QIAGEN). cDNA synthesis was performed using iScript Reverse Transcription Supermix for RT-PCR (iScript Reverse Transcription Supermix, Bio-Rad) according to the manufacturer's instructions using the following protocol: $5 \mathrm{~min}$ at $25^{\circ} \mathrm{C}, 20 \mathrm{~min} 46{ }^{\circ} \mathrm{C}, 1$ min $95^{\circ} \mathrm{C}$. PCR reactions were carried out using 96-well plates on an Applied Biosystems ViiA 7 Real-Time PCR System using the following parameters: $95^{\circ} \mathrm{C}$ for $20 \mathrm{~s}, 40$ cycles of $95^{\circ} \mathrm{C}$ for 1 $\mathrm{s}$, and $60^{\circ} \mathrm{C}$ for $20 \mathrm{~s}$. The final volume $(10 \mu \mathrm{l})$ of PCR SYBR-Green reaction consisted of $5 \mu$ fast SYBR-Green Master Mix (Applied Biosystems), $2 \mu \mathrm{l}$ cDNA, $1 \mu \mathrm{l}$ of $5 \mu \mathrm{M}$ forward and reverse primers, and $1 \mu \mathrm{l}$ of water. 


\begin{tabular}{|c|c|c|}
\hline $\begin{array}{l}\text { Gene } \\
\text { symbol }\end{array}$ & Forward & Reverse \\
\hline $18 s$ & AGTCCCTGCCCTTTGTACACA & CGATCCGAGGGCCTCACTA \\
\hline Acc1 & AATGAACGTGCAATCCGATTTG & ACTCCACATTTGCGTAATTGTTG \\
\hline Acc2 & CGCTCACCAACAGTAAGGTGG & GCTTGGCAGGGAGTTCCTC \\
\hline Acly & AATCCTGGCTAAAACCTCGCC & GCATAGATGCACACGTAGAACT \\
\hline Actin & GGCTGTATTCCCTCCATCG & CCAGTTGGTAACAATGCCATGT \\
\hline Aldh1/1 & AGCCACCTATGAGGGCATTC & TGAGTGTCGAGTTGAAAAACGTC \\
\hline Aldh1/2 & ACCAGCCGGGTTTATTTTCAAA & ACTCCCACTACTCGGTGGC \\
\hline Dgat1 & CTGATCCTGAGTAATGCAAGGTT & TGGATGCAATAATCACGCATGG \\
\hline Dgat2 & GCGCTACTTCCGAGACTACTT & GGGCCTTATGCCAGGAAACT \\
\hline Dhcr7 & AGGCTGGATCTCAAGGACAAT & GCCAGACTAGCATGGCCTG \\
\hline Dhcr24 & CTCTGGGTGCGAGTGAAGG & TTCCCGGACCTGTTTCTGGAT \\
\hline Dld & GAGCTGGAGTCGTGTGTACC & GAACCTATCACTGTCACGTCA \\
\hline Fasn & GGAGGTGGTGATAGCCGGTAT & TGGGTAATCCATAGAGCCCAG \\
\hline Fdft1 & GTTTGAAGACCCCATAGTTGGTG & CACATCTACGTTCTCTGGCTTAG \\
\hline Fdps & GGAGGTCCTAGAGTACAATGCC & AAGCCTGGAGCAGTTCTACAC \\
\hline Ggps1 & TTCACAGGCATTTAATCACTGGC & ACCACGTCGGAGCTTTGAAC \\
\hline Gldc & CTCCTGCCCAGACACGAT & GGGACCGTCTTCTCGATGAG \\
\hline Gpat1 & CTTGGCCGATGTAAACACACC & CTTCCGGCTCATAAGGCTCTC \\
\hline Gpat4 & AGAAATTCGTCGAAGTGGT & CCTTTCCGGCAAAAGTAGAAGAT \\
\hline Hmgcs1 & AACTGGTGCAGAAATCTCTAGC & GGTTGAATAGCTCAGAACTAGCC \\
\hline $\mathrm{Hmgcr}$ & AGCTTGCCCGAATTGTATGTG & TCTGTTGTGAACCATGTGACTTC \\
\hline Lss & TAAAAC & CGTCCTCCGCTTGATAATAAGTC \\
\hline Mthfd1 & CCCAAGTGACATTG & TAGCCTTCGTTTCCCCGTACA \\
\hline Mthfd2 & AATGAAGCCGTTG & GACTGGCGGGATTGTCACC \\
\hline Mthfd11 & GAT & GTACGAGCTTCCC \\
\hline Mthfd2I & ACGTTGATGGATTTCACAT & GATGATTTCCCAAACGGCACT \\
\hline Mthfr & AGGCGCAGAATGGAC & CATCCGGTCAAACCTGGAGAT \\
\hline Mtr & TCCTCCTCGGCCTATCTTTATTT & GGTCCGAATGAGACACGCT \\
\hline Mvk & GGTGTGGTCGGAACTTCCC & CCTTGAGCGGGTTGGAGAC \\
\hline Mvd & CTCAGAAAAGCCTCAG & TGGTCGTTTTTAGCTGGTCCT \\
\hline Pmvk & CCTATGGGGCTGTGATACAGA & TCTCCGTGGTTCTCAATGACC \\
\hline Psat & CAGTGGAGCGCCAGAATAGAA & CCTGTGCCCCTTCAAGGA \\
\hline Psph & TGAGTACGCAGGTTTTTATGAG & TGAGTACGCAGGTTTTGATGAG \\
\hline Phgdh & ATGGCCTTCGCAAATCTGC & AGTTCAGCTATCAGCTCCTCC \\
\hline Scd1 & TTCTTGCGATACACTCTGGTGC & CGGGATTGAATGTTCTTGTCGT \\
\hline Scd2 & GATCTCTGGCGCTTACTCAGC & CTCCCCAGTGGTGAGAACTC \\
\hline Sds & GAAGACCCCACTTCGTGACAG & TCTTGCAGAGATGCCCAATGC \\
\hline Shmt1 & CAGGGCTCTGCTTGATGCAC & CGTAACGCGCTCTTGTCAC \\
\hline Shmt2 & TGGCAAGAGATACTACGGAGG & AGATCCGCTTGACATCAGACA \\
\hline Sqle & ATAAGAAATGCGGGGATGTCAC & ATATCCGAGAAGGCAGCGAAC \\
\hline Srebp1a & $\begin{array}{l}\text { TAGTCCGAAGCCGGGTGGGCGC } \\
\text { CGG }\end{array}$ & $\begin{array}{l}\text { GATGTCGTTCAAAACCGCTGTGT } \\
\text { GTC }\end{array}$ \\
\hline Srebp1c & AAGCAAATCACTGAAGGACCTGG & AAAGACAAGCTACTCTGGGAG \\
\hline Srebp2 & GGATCCTCCCAAAGAAGGAG & TTCCTCAGAACGCCAGACTT \\
\hline Tyms & GGAAGGGTGTTTTGGAGGAGT & GCTGTCCAGAAAATCTCGGGA \\
\hline
\end{tabular}




\section{Western blotting}

To compare tissue protein levels, $\sim 20 \mathrm{mg}$ of tissue was homogenized in RIPA buffer supplemented with $5 \mathrm{mM}$ EDTA solution (Thermo Scientific), protease inhibitor cocktail (cOmplete, Roche) and phosphatase inhibitor cocktail (PhosSTOP, Roche), and placed on ice for $30 \mathrm{~min}$. Tissue lysate was centrifuged at $13000 \mathrm{~g}$ for $10 \mathrm{~min}$ at $4{ }^{\circ} \mathrm{C}$, and the supernatant was stored at $-80^{\circ} \mathrm{C}$. Homogenate protein content was determined using the bicinchoninic acid assay (Thermo Scientific). Protein samples were prepared in a Laemmli buffer (NuPAGE LDS Sample Buffer, Life Technologies), and were run a 4-15\% precast gel (Mini-PROTEAN TGX, Bio-Rad) for 2 hours at constant $100 \mathrm{~V}$ and transferred on a polyvinylidenedifluoride (PVDF) membrane for 2 hours at constant $250 \mathrm{~mA}$ in an ice-chilled transfer tank. The membrane was blocked with $5 \%$ milk and incubated overnight at $4^{\circ} \mathrm{C}$ with a primary antibody against ACLY (Cell Signaling \#13390), ACC (Cell Signaling \#3662), P-Akt ${ }^{\text {Ser473 }}$ (Cell Signaling \#9271), P-Akt ${ }^{\text {Ser308 }}$ (Cell Signaling \#9275), Akt (Cell Signaling \# 75692), SCD1 (Cell Signaling \#2794), GAPDH (Cell Signaling \#5174), and vinculin (Cell Signaling \#4650). After washing with TBS-T, membranes were incubated with a horseradish peroxidase (HRP)-conjugated secondary antibody for 1 hour at room temperature and incubated with enhanced chemiluminescence liquid (Clarity Western ECL Substrate, Bio-Rad) for 5 min. Densitometry quantification was performed using Image Lab software (Bio-Rad). 


\section{Figures}

\section{Figure 1}

See figure for legend.

\section{Figure 2}

See figure for legend.

\section{Figure 3}

See figure for legend.

\section{Figure 4}

See figure for legend.

\section{Supplementary Files}

This is a list of supplementary files associated with this preprint. Click to download.

- SupplementaryTable1.Dietcomposition.pdf

- SupplementaryTable2.LCMS.pdf

- SupplementaryTable3.QE.pdf

- ExtendedData.pdf 